\title{
Internal solitary waves propagating through variable background hydrology and currents
}

\author{
Z. Liu , R. Grimshaw and E. Johnson \\ Department of Mathematics, University College London, UK
}

\begin{abstract}
Large-amplitude, horizontally-propagating internal wave trains are commonly observed in the coastal ocean, fjords and straits. They are long nonlinear waves and hence can be modelled by equations of the Korteweg-de Vries type. However, typically they propagate through regions of variable background hydrology and currents, and over variable bottom topography. Hence a variable-coefficient Korteweg-de Vries equation is needed to model these waves. Although this equation is now well-known and heavily used, a term representing non-conservative effects, arising from dissipative or forcing terms in the underlying basic state, has usually been omitted. In particular this term arises when the hydrology varies in the horizontal direction. Our purpose in this paper is to examine the possible significance of this term. This is achieved through analysis and numerical simulations, using both a two-layer fluid model and a re-examination of previous studies of some specific ocean cases.
\end{abstract}

Keywords: Internal Solitary Waves, Korteweg-de Vries Equation

\section{Introduction}

Large amplitude internal solitary wave are commonly observed in the coastal ocean, fjords and straits, see the reviews by Grimshaw (2001), Holloway et al. (2001), Ostrovsky and Stepanyants (2005), Helfrich and Melville (2006), Grimshaw (2007), Grimshaw et al. (2010) and the book by Vlasenko et al. (2005). They are long waves with wavelengths greater than the relevant vertical scale (such as the fluid depth or pycnocline depth) and hence it is now widely accepted that the basic paradigm for the description of these waves is based on the Korteweg-de Vries (KdV) equation, first derived in this 
context by Benney (1966) and Benjamin (1966) and subsequently by many others, see the aforementioned references.

However, typically they propagate through regions of variable hydrology and currents, and over variable bottom topography. Hence a variablecoefficient Korteweg-de Vries (vKdV) equation has been commonly used to model these waves. For internal waves this is given by, see Grimshaw (1981); Zhou and Grimshaw (1989) for a detailed derivation,

$$
\eta_{t}+c \eta_{x}+\frac{c Q_{x}}{2 Q} \eta+\mu \eta \eta_{x}+\delta \eta_{x x x}+\sigma \eta=0
$$

Here $\eta(x, t)$ is the amplitude of the wave elevation above the undisturbed level, see (17) below, $x, t$ are space and time variables respectively, and subscripts denote derivatives. Here $c(x)$ is the relevant linear long wave speed, and $Q(x)$ is the linear magnification factor, defined so that $Q \eta^{2}$ is the wave action flux density for linear long waves. The coefficients $c, Q, \mu, \delta, \sigma$ are determined by the waveguide properties of the specific physical system being considered, and they are slowly-varying functions of $x$. Our concern in this paper is especially with the final term $\sigma \eta$ which in general is due to nonconservative effects arising from dissipative or forcing terms in the underlying basic state, see Andrews and McIntyre (1978); Grimshaw (1984) for a general discussion of how this term arises when considering wave action conservation. We use the term "non-conservative" here and throughout advisedly in the restricted sense that the term is non-conservative only within the framework of the chosen basic equation set. A term of this form representing dissipation effects on solitary waves was first introduced by Ott and Sudan (1970), but for the case when there is no background inhomogeneity and so, in (1), the coefficients $c, \mu, \delta, \sigma, Q$ are each constant. In the present internal wave context, this term was first derived by Pelinovsky et al. (1977) for the special case when the bottom is flat, there is no background current and nonconservative effects arise solely to a horizontally varying background density field. Later Grimshaw (1981) derived this term in the general case when the bottom topography, the background current and the background density field may all vary in the horizontal direction, see also Zhou and Grimshaw (1989) for a simplified description of the derivation. More recently Vlasenko and Stashchuk (2006) derived a wave energy equation with a source term describing the interaction with the background flow field, for the special case of internal waves interacting with a barotropic tidal current with horizontal shear arising due to a bottom topographic slope. However, under the Boussi- 
nesq approximation used by Vlasenko and Stashchuk (2006) the system is conservative, and we assume that the derived wave energy equation can be expressed as a conservative wave action equation. Although the term $\sigma \eta$ in (1) has been known for over three decades, it has usually been neglected in modelling studies of internal waves. Our purpose here is to rectify that omission and test the possible significance of this term.

A brief summary of the derivation of (1) is given in section 2, emphasising the origin of the term $\sigma \eta$. Here we first note that the derivation uses the usual KdV balance that the weak nonlinearity has the same order as the weak linear dispersion, that is $\eta \eta_{x}$ has the same order of magnitude as $\eta_{x x x}$, and in addition assumes that the waveguide properties (that is, the coefficients $c, Q, \mu, \delta, \sigma)$ vary slowly so that both $\eta Q_{x} / Q$ and $\sigma \eta$ are of the same order of magnitude. In this scenario, the first two terms in (1) are the dominant terms, and hence we can make the transformation

$$
A=\sqrt{Q} \eta, \quad T=\int_{0}^{x} \frac{d x}{c}, \quad X=T-t .
$$

Substitution into (1) yields, to the same order of approximation as in the derivation of (1),

$$
\begin{gathered}
A_{T}+\nu A A_{X}+\lambda A_{X X X}+\sigma A=0, \\
\nu=\frac{\mu}{c \sqrt{Q}}, \quad \lambda=\frac{\delta}{c^{3}} .
\end{gathered}
$$

The coefficients $\nu, \lambda, \sigma$ are functions of $T$ alone. Note that although $T$ is a variable along the spatial path of the wave, we shall subsequently refer to it as the "time". Similarly, although $X$ is a temporal variable (in a reference frame moving with speed $c$ ), we shall subsequently refer to it as a "space" variable. Finally, since we can assume $\delta>0$ in practice see (21) below for right-going waves, it is useful to make a further transformation yielding the canonical form,

$$
\begin{gathered}
A_{\tau}+\alpha A A_{X}+A_{X X X}+\beta A=0 . \\
\text { where } \tau=\int_{0}^{T} \lambda d T=\int_{0}^{x} \frac{\delta}{c^{4}} d x, \quad \alpha=\frac{\nu}{\lambda}=\frac{\mu c^{2}}{\sqrt{Q} \delta}, \quad \beta=\frac{\sigma}{\lambda}=\frac{\sigma c^{3}}{\delta} .
\end{gathered}
$$

Note that the coefficients $\alpha, \beta$, originally expressed as functions of $x$, are now functions of $\tau$ through the transformation $\tau=\tau(x)$ in (6). 
We shall call equation (5) the vKdV equation. It has two conservation laws

$$
\begin{aligned}
& \frac{\partial M}{\partial \tau}=-\beta M, \quad M=\int_{-\infty}^{\infty} A d X, \\
& \frac{\partial P}{\partial \tau}=-2 \beta P, \quad P=\int_{-\infty}^{\infty} A^{2} d X,
\end{aligned}
$$

for mass and wave action flux respectively, that express the conservation of the transformation $M, P$ in the conservative case when $\beta=0$. Note that

$$
A=R B, \quad R=\exp \left(-\int_{0}^{\tau} \beta d \tau^{\prime}\right), \quad B_{\tau}+R \alpha B B_{X}+B_{X X X}=0,
$$

leads to the usual vKdV equation but with a modified nonlinear coefficient. This transformed equation is useful for numerical simulations, where we simulate this version with a pseudo-spectral method and fourth-order RungeKutta for the time-discretization. Note that the scaling factor $R$ is a cumulative measure of the coefficient $\beta$.

In section 2 we present a brief summary of the derivation of (1) emphasising the origin of the coefficient $\sigma$. Then in sections 3 and 4 we present some analysis and numerical simulations to examine the possible significance of this coefficient. In section 3 we use a two-layer fluid model for two cases; one where there is horizontal variation in the hydrology but any background current is ignored, and the other where the horizontal variation in the hydrology is supported by a background current. In section 4 we re-examine three ocean cases studied by Grimshaw et al. (2004) where there may have been horizontal variation in hydrology, but the term $\sigma \eta$ in (1) was ignored. We conclude in section 5 .

\section{Variable-coefficient Kortweg-de Vries equation}

A derivation of the $\mathrm{vKdV}$ equation (1) is briefly reproduced here to bring out the origin of the coefficient $\sigma$. In the ocean, the background state varies slowly in the horizontal due to varying depth, and slow variations in the basic state hydrology and background currents. That is, the depth $h=$ $h(x)$, the background horizontal current $u_{0}=u_{0}(z ; x)$ with a corresponding vertical velocity field $w_{0}(z, x)$, a density field $\rho_{0}(z ; x)$ a corresponding pressure field $p_{0}(z ; x)$ and a free surface displacement $\eta_{0}(x)$. This basic state satisfies 
the full steady-state Euler equations, with the exception of the momentum equations where there are body forces $F_{0}(z ; x), G_{0}(z ; x)$ respectively. That is

$$
\begin{aligned}
\rho_{0}\left(u_{0} u_{0 x}+w_{0} u_{0 z}\right)+p_{0 x} & =F_{0}, \\
{\left[\rho_{0}\left(u_{0} w_{0 x}+w_{0} w_{0 z}\right)\right]+p_{0 z}+g \rho_{0} } & =\left[G_{0}\right], \\
u_{0} \rho_{0 x}+w_{0} \rho_{0 z} & =0, \\
u_{0 x}+w_{0 z} & =0, \\
w_{0}+u_{0} h_{x} & =0, \text { at } z=-h(x), \\
p_{0} & =0, \text { at } z=\eta_{0}, \\
u_{0} \eta_{0 x} & =w_{0}, \text { at } z=\eta_{0} .
\end{aligned}
$$

Because the $x$-dependence is slow, technically $\partial / \partial x \sim \epsilon^{3}, w_{0} \sim \epsilon^{3}, F_{0} \sim \epsilon^{3}$ and $G_{0} \sim \epsilon^{6}$, the dominant balance in the vertical momentum equation is hydrostatic and the terms in [.] can be omitted. Thus, of most importance here, the horizontal body force $F_{0}(z ; x)$ represents terms not present in the left-hand side of (10) such as diabatic effects, for instance dissipation, Corlolis terms, and driving terms, for instance wind stress. In this paper, we do not examine this issue directly. Instead, we use model or observed background fields $\rho_{0}(z ; x)$ and $u_{0}(z ; x)$ to estimate the body force $F_{0}(z ; x)$ directly, and hence $\sigma$.

This basic state is then perturbed by the wave field, where at the leading order the vertical particle displacement is given by

$$
\zeta \sim \eta(x, t) \phi(z, x)
$$

Here the modal function $\phi(z ; x)$ together with the linear long wave speed $c(x)$ is defined by the boundary-value problem,

$$
\begin{aligned}
\left\{\rho_{0}\left(c-u_{0}\right)^{2} \phi_{z}\right\}_{z}-g \rho_{0 z} \phi & =0, \quad \text { for }-h<z<\eta_{0}, \\
\phi=0 \quad \text { at } z=-h, \quad\left(c-u_{0}\right)^{2} \phi_{z} & =g \phi \quad \text { at } z=\eta_{0} .
\end{aligned}
$$

Here the $x$-dependence is parametric, and is slowly-varying. Continuation of this asymptotic expansion to the next order than yields the vKdV equation (1), see Pelinovsky et al. (1977); Grimshaw (1981); Zhou and Grimshaw (1989) or the reviews by Grimshaw (2007); Grimshaw et al. (2010). The 
coefficients are given by

$$
\begin{aligned}
I \mu & =3 \int_{-h}^{\eta_{0}} \rho_{0}\left(c-u_{0}\right)^{2} \phi_{z}^{3} d z \\
I \delta & =\int_{-h}^{\eta_{0}} \rho_{0}\left(c-u_{0}\right)^{2} \phi^{2} d z \\
\text { where } I & =2 \int_{-h}^{\eta_{0}} \rho_{0}\left(c-u_{0}\right) \phi_{z}^{2} d z \\
\text { and } \quad Q & =c^{2} I \Delta, \quad I \sigma=-\int_{-h}^{\eta_{0}} \phi \phi_{z} F_{0 z} d z .
\end{aligned}
$$

These expressions are well-known, except for the expression $\Delta$ and the coefficient $\sigma . \Delta$ represents wave spreading; for instance in a cylindrical geometry where $x$ is a radial coordinate, $\Delta=x$ where the virtual source is at $x=0$. In the one-dimensional case considered here, $\Delta=1$. The coefficient $\sigma$ represents non-conservative effects, and was derived in this form by Grimshaw (1981). The same term was derived earlier by Pelinovsky et al. (1977) for the special case when the bottom was flat and there is no background current. The derivation can also be obtained directly from the general theory for wave action, see Andrews and McIntyre (1978); Grimshaw (1984).

It is necessary to express all variables and coefficients in non-dimensional variables based on a length scale $h_{0}$, a typical depth, and a velocity scale $c_{0}$, a typical linear long wave speed, so that the time scale is $t_{0}=h_{0} / c_{0}$. For instance, in an ocean, setting $h_{0}=100 \mathrm{~m}$ as a typical thermocline depth and $c_{0}=1 \mathrm{~m} \mathrm{~s}^{-1}$ as a typical speed. If needed the density $\rho_{0}$ can be scaled with $\rho_{00}=1 \mathrm{~kg} \mathrm{~m}^{-3}$. The modal function is non-dimensional with maximum amplitude 1. Formally, we write

$$
\begin{aligned}
\eta=h_{0} \bar{\eta}, \quad x=h_{0} \bar{x}, \quad t=\frac{h_{0} \bar{t}}{c_{0}} \\
(c, U)=c_{0}(\bar{c}, \bar{U}), \quad \mu=\frac{c_{0} \bar{\mu}}{h_{0}}, \quad \delta=c_{0} h_{0}^{2} \bar{\delta}, \quad \sigma=c_{0} \bar{\sigma} .
\end{aligned}
$$

Then the $\mathrm{KdV}$ equation (1) is recovered in the non-dimensional variables, and also all expressions $(20-23)$ hold in the non-dimensional variables. Henceforth non-dimensional is omitted.

We conclude this section with a brief summary of modulated periodic and solitary wave solutions, see the recent account by Grimshaw and Yuan (2016) and the references therein. When the coefficient $\alpha$ in (5) is a constant 
the $\mathrm{KdV}$ equation supports a periodic travelling wave, $A(X-V \tau)$, the wellknown cnoidal wave solution

$$
\begin{gathered}
A=a\left\{b(m)+\mathrm{cn}^{2}(\gamma \theta ; m)\right\}+d, \quad \theta=k(X-V \tau), \\
\text { where } \alpha a=12 m \gamma^{2} k^{2}, \quad b(m)=\frac{1-m}{m}-\frac{E(m)}{m K(m)}, \\
V-\alpha d=\frac{\alpha a}{3}\left\{\frac{2-m}{m}-\frac{3 E(m)}{m K(m)}\right\}=4 \gamma^{2} k^{2}\left\{2-m-\frac{3 E(m)}{K(m)}\right\} .
\end{gathered}
$$

Here $c n(x ; m)$ is the Jacobian elliptic function of modulus $m, 0<m<1$, and $K(m)$ and $E(m)$ are the elliptic integrals of the first and second kind. The expression (25) has period $2 \pi$ in $\theta$ so that $\gamma=K(m) / \pi$, while the spatial period is $2 \pi / k$. The (trough-to-crest) amplitude is $a$ and the mean value over one period is $d$. It is a three-parameter family with parameters $k, m, d$ say. As the modulus $m \rightarrow 1$, this becomes a solitary wave, since then $b \rightarrow 0$ and $\operatorname{cn}(x) \rightarrow \operatorname{sech}(x)$, while $\gamma \rightarrow \infty, k \rightarrow 0$ with $\gamma k=\Gamma$ fixed. As $m \rightarrow 0$, $b \rightarrow-1 / 2, \gamma \rightarrow 1 / 2, \operatorname{cn}(x) \rightarrow \cos (x)$, and it reduces to a sinusoidal wave $(a / 2) \cos (\theta)$ of small amplitude $a \sim m$ and wavenumber $k$.

We now allow this cnoidal wave to vary slowly with $\tau$; that is, the parameters $k, m, d$ vary slowly with $\tau$. As three modulation equations are needed, we supplement $(7,8)$ with the equation for conservation of waves,

$$
k_{\tau}+(k V)_{X}=0 .
$$

Since here we are allowing only $\tau$-modulations, it follows that $k$ is a constant. The remaining two modulation equations are obtained by inserting the cnoidal wave solution into the conservation laws $(7,8)$ and averaging over the phase $\theta$. The outcomes are

$$
\begin{gathered}
d_{\tau}=-\beta d, \\
\mathcal{P}_{\tau}=-2 \beta \mathcal{P}, \quad \mathcal{P}=<A^{2}>,
\end{gathered}
$$

where the $\langle\cdots\rangle$ denotes a $2 \pi$-average over $\theta$. The expression $\mathcal{P}$ is given by

$$
\begin{aligned}
\mathcal{P} & =d^{2}+a^{2}\left\{C_{4}-b^{2}\right\} \\
C_{4} & =\frac{1}{3 m^{2} K(m)}\left\{3 m^{2} K(m)-5 m K(m)+4 m E(m)+2 K(m)-2 E(m)\right\} .
\end{aligned}
$$


Note that the equation for the mean level (29) is uncoupled from (30) and can be solved independently.

In the solitary wave limit $m \rightarrow 1$ and then $b \sim-1 / K(m) \rightarrow 0$ and $C_{4} \sim 2 / 3 K(m) \rightarrow 0$. The cnoidal wave expression (25) becomes

$$
\left.A=a \operatorname{sech}^{2}(\gamma \theta)\right\}+d, \quad \theta=k(X-V \tau), \quad V-\alpha d=\frac{\alpha a}{3}=12 \gamma^{2} k^{2},
$$

with one parameter to be determined. This is obtained from a reduction of (30) as $m \rightarrow 1$, or more directly by averaging the wave action conservation law (8) directly for a solitary wave, see Grimshaw (1979) and the discussion in El et al. (2012). Setting $d=0$ and using the expressions in (32), (33) this becomes

$$
\begin{gathered}
\mathcal{A}_{\tau}=-2 \beta \mathcal{A}, \quad \mathcal{A}=\left\{\frac{a^{3}}{\alpha}\right\}^{1 / 2}, \\
\frac{a^{3}}{a_{0}^{3}}=\frac{\alpha}{\alpha_{0}} \exp \left(-4 \int_{0}^{\tau} \beta d \tau\right),
\end{gathered}
$$

where the "0" subscript denotes the value at $\tau=0$. In terms of the original $\mathrm{KdV}$ equation (1) the amplitude of the solitary wave is $a_{s}=a Q^{-1 / 2}$ and taking account of (4), (34) becomes

$$
\frac{a_{s}^{3}}{a_{s 0}^{3}}=\frac{\kappa}{\kappa_{0}} \exp \left(-4 \int_{0}^{T} \sigma d T\right), \quad \kappa=\frac{c^{2} \mu}{Q^{2} \delta} .
$$

The case of a periodic wave modulated only in $\tau$ was studied by Grimshaw $(2007,2015)$ and is reproduced briefly here. For simplicity we again set $d=0$ and then, using the expressions in (26), (30) becomes

$$
\frac{F(m)}{F\left(m_{0}\right)}=\frac{\alpha^{2}}{\alpha_{0}^{2}} \exp \left(-2 \int_{0}^{\tau} \beta d \tau\right),
$$

where $F(m)=K(m)^{2}\left\{(4-2 m) E(m) K(m)-3 E(m)^{2}-(1-m) K(m)^{2}\right\}$.

We see that when $\beta=0$, then as $|\alpha|$ increases/decreases, so does the modulus $m$.

\section{Applications I}

In this section we examine some analytical models of the effect of a horizontal variation of the background hydrology and current. 


\subsection{Horizontal variation only in hydrology}

For this application, we assume that the basic current $u_{0}(z ; x)=0$, but that the density profile $\rho_{0}(z ; x)$ depends on both $z$ and $x$, where the latter dependence is slowly varying. Then the basic state equations $(10-16)$ reduce to

$$
p_{0 x}=F_{0}, \quad p_{0 z}+g \rho_{0}=0, \quad \text { and } p_{0}=0 \quad \text { at } \quad z=0 .
$$

Note that here we can set the free surface displacement $\eta_{0}=0$. It follows that, using (23)

$$
F_{0 z}=-g \rho_{0 x}, \quad I \sigma=\int_{-h}^{0} \phi \phi_{z} g \rho_{0 x} d z=\left[\frac{1}{2} g \rho_{0 x} \phi^{2}\right](z=0)-\frac{1}{2} \int_{-h}^{0} g \rho_{0 z x} \phi^{2} d z
$$

and so the non-conservative term $\sigma$ is determined by the horizontal gradient of the basic density profile $\rho_{0}(z ; x)$. In the Boussinesq approximation for internal waves the inertial effects of the density can be ignored; then it follows in particular that the modal function $\phi=0$ at $z=0$ and so the first term in (39) can be ignored. It follows that $\sigma$ is positive or negative depending on whether the stratification is increasing or decreasing in the horizontal direction. For instance on the Australian North West Shelf, the stratification decreases towards the coast, see figure 4 of Grimshaw et al. (2006), implying that $\beta<0$ and hence causing wave amplification.

It is useful to note that from the modal equation (18) and the definition of $I, Q(22,23)$ that in the absence of the background current,

$$
\frac{c I}{2}=c^{2} \int_{-h}^{0} \rho_{0} \phi_{z}^{2} d z=\left[g \rho_{0} \phi^{2}\right](z=0)-\int_{-h}^{0} g \rho_{0 z} \phi^{2} d z .
$$

Then, on differentiating with respect to $x$ and using (39), we get that

$$
\sigma=\frac{c_{x}}{2}+\frac{1}{2 I}\left[\rho_{0} \phi_{z}^{2} h_{x}\right](z=-h)+\frac{1}{2 I} \int_{-h}^{0} \rho_{0 x} \phi_{z}^{2} d z .
$$

The second term is due to the specific dependence of $c$ on the depth $h$,

$$
\sigma=\frac{c_{x}}{2}-\frac{c_{h} h_{x}}{2}+\frac{1}{2 I} \int_{-h}^{0} \rho_{0 x} \phi_{z}^{2} d z=\frac{\left[c_{x}\right](h=\text { constant })}{2}+\frac{1}{2 I} \int_{-h}^{0} \rho_{0 x} \phi_{z}^{2} d z .
$$

The first term is thus the horizontal variation in the speed $c$ due to the density stratification alone, and the last term can be omitted in the Boussinesq 
approximation. Thus, in the Boussinesq approximation, $\sigma>0(<0)$ and the wave decays (amplifies) due to this term according as $\left[c_{x}\right](h=$ constant $)>$ $0(<0)$, that is as the speed $c$ increases or decreases with the stratification. Since the modal equation (18) implies that for an internal wave $c$ scales with $N h$, in general we expect $c$ to increase or decrease as the stratification increases or decreases, and hence then $\sigma>0(<0)$ accordingly. Note that the last term in the expression (59) for $\sigma$, neglected in the Boussinesq approximation, has the same effect.

As a specific example, choose a two-layer fluid, where the density profile is

$$
\rho_{0}=\left(\rho_{00}-\frac{\Delta \rho}{2}\right) H\left(z+h_{1}\right)+\left(\rho_{00}+\frac{\Delta \rho}{2}\right) H\left(-z-h_{1}\right),
$$

where $H(\cdots)$ is the usual Heaviside function and $h_{1,2}$ are the depths of the upper and lower layers, $h_{1}+h_{2}=h$. In the Boussinesq approximation, the modal function and linear long wave speed are given by

$$
\phi=-\frac{z}{h_{1}} H\left(z+h_{1}\right)+\frac{z+h}{h_{2}} H\left(-z-h_{1}\right), \quad c^{2}=\frac{g^{\prime} h_{1} h_{2}}{h}, \quad g^{\prime}=\frac{g \Delta \rho}{\rho_{00}} .
$$

The coefficients $(20-23)$ are then

$$
\mu=\frac{3 c\left(h_{1}-h_{2}\right)}{2 h_{1} h_{2}}, \quad \delta=\frac{c h_{1} h_{2}}{6}, \quad I=\frac{2 c h}{h_{1} h_{2}}, \quad Q=c^{2} I .
$$

Here we have absorbed the constant $\rho_{00}$ into $I$, that is, we have replaced $I$ with $I / \rho_{00}$. To find an expression for $\sigma$ we assume that both $\Delta \rho$ and $h_{1}$ vary with $x$, corresponding to changing stratification and changing pycnocline depth respectively, so that then the coefficients (45) also vary with $x$. Then, from (39),

$$
I \sigma=\frac{g_{x}^{\prime}}{2}-\frac{g^{\prime} h_{1 x}\left(h_{1}-h_{2}\right)}{2 h_{1} h_{2}}, \quad \text { so that } \quad \sigma=\frac{1}{2}\left[c_{x}\right](h=\text { constant }),
$$

in agreement with the general result (59). Thus $\sigma$ is positive or negative according as either $g_{x}^{\prime}$ is positive or negative, or as $h_{1 x}\left(h_{1}-h_{2}\right)$ is positive or negative. Thus, a shallowing pycnocline, $h_{1 x}>0, h_{1}<h_{2}$ with weaker stratification, $g_{x}^{\prime}<0$, leads to $\sigma<0$ and hence wave amplification. For instance suppose that $c$ decreases from $2 \mathrm{~ms}^{-1}$ to $1 \mathrm{~ms}^{-1}$ uniformly over a horizontal distance of $100 \mathrm{~km}$; then $\sigma \sim-0.5 \times 10^{-5} \mathrm{~s}^{-1}$ in dimensional terms. 
As a specific oceanic example, we choose $h=1000-500 \chi m$, and $h_{1}=$ $200-150 \chi m$, where $\chi=0.5 \times 10^{-5}$ in the range $0<\chi<1$. This corresponds to a shelf of width $200 \mathrm{~km}$, that is $0<x<200 \mathrm{~km}$, where the depth decreases from $h=1000 \mathrm{~m}$ to $h=500 \mathrm{~m}$, the upper layer depth decreases from $h_{1}=$ $200 \mathrm{~m}$ to $h_{1}=50 \mathrm{~m}$, and then the lower layer depth decreases from $h_{2}=$ $800 \mathrm{~m}$ to $h_{2}=450 \mathrm{~m}$. The coefficients $\mu, \delta, Q$ can then be computed from (45) and note in particular that $\mu<0$ throughout. Then we also suppose that the stratification increases shorewards so that $g^{\prime}=0.01+0.01 \chi \mathrm{ms}^{-2}$, from which $\sigma$ can be found from (46). These coefficients are shown in figure 1. The first term in $\sigma$ due to $g_{x}^{\prime}$ is positive but the second term due to $h_{1 x}$ is negative. The combination is initially small and positive, but soon becomes negative as the second term dominates as $h_{1}$ decreases. This can also be seen in the plot for the scaling factor $R$ (9) which is initially close to unity, but then significantly increases. A simulation of the $\mathrm{vKdV}$ equation (5) is shown in figure 2. Here $\alpha<0$ throughout and increases in magnitude, leading to amplitude growth, and incipient fissioning. The effect of the term with coefficient $\sigma$ is to enhance the nonlinearity sufficiently that the leading wave increases further in amplitude and enhances the fissioning

\subsection{Current with only horizontal shear}

For this application we assume that the basic current $u_{0}(z ; x)=U(x)$ does not depend on $z$, but the density profile $\rho_{0}(z ; x)$ depends on both $z$ and $x$, where the latter dependence is slowly varying. This situation was considered by Vlasenko and Stashchuk (2006) in a study of the effect of a background barotropic tidal current with horizontal shear on the internal waves generated by the tidal flow over a sill. They combined a theoretical analysis of the interaction between the waves and the background tidal flow with numerical simulations, using the fully nonlinear equations in a Boussinesq approximation, to study the well-known generation of internal solitary waves in Knight Inlet. Their main conclusion is that these internal waves are amplified when propagating either upstream into a region of increasing current, or downstream into a region of decreasing current; otherwise the waves are suppressed. Here we examine this same situation but within the framework of the vKdV equation (1). In this special case the basic state 
equations $(10-16)$ reduce to

$$
\begin{aligned}
& \rho_{0} U U_{x}+p_{0 x}=F_{0} \\
& p_{0 z}+g \rho_{0}=0 \\
& u_{0}=\psi_{0 z}, \quad w_{0}=-\psi_{0 x} \\
& \psi_{0}=M_{0} y, \quad y=\frac{z+h}{H}, \quad \psi_{0}\left(z=\eta_{0}\right)=H U=M_{0}, \quad H=h+\eta_{0} \\
& \rho_{0}=\rho_{0}(y)
\end{aligned}
$$

It follows that

$$
\begin{gathered}
F_{0 z}=\rho_{0 z} U U_{x}-g \rho_{0 x}, \quad U U_{x}=-U^{2} \frac{H_{x}}{H}, \\
\rho_{0 x}=-\frac{w_{0} \rho_{0 z}}{U}=\rho_{0 z}\left(H_{x}(1-y)-\eta_{0 x}\right)
\end{gathered}
$$

The modal function now satisfies $(18,19)$, which can be rewritten in the scaled form using the change of variable $y=(z+h) / H$,

$$
\begin{aligned}
& \tilde{c}^{2}\left(\rho_{0} \phi_{y}\right)_{y}-\rho_{0 y} \phi=0, \quad \tilde{c}=\frac{\hat{c}}{c_{0}} \quad \text { for } \quad 0<y<1, \\
& \phi=0 \quad \text { at } \quad y=0, \quad \tilde{c}^{2} \phi_{y}=\phi \quad \text { at } \quad y=1 .
\end{aligned}
$$

Here $\hat{c}=c-U$ and $c_{0}=(g H)^{1 / 2}$, and $\rho_{0}=\rho_{0}\left(M_{0} y\right)$ depends only on $y$. In this scaled form there is no explicit $x$-dependence in the modal equation, and hence $\phi(y)$ and $\tilde{c}$ have no explicit $x$-dependence. Using the same scaling the expressions $(20-23)$ for the coefficients become

$$
\begin{aligned}
\mu & =\frac{3 c_{0}}{2 H} \tilde{\mu}, \quad \tilde{I} \tilde{\mu}=\tilde{c} \int_{0}^{1} \rho_{0} \phi_{y}^{3} d y \\
\delta & =\frac{c_{0} H^{2}}{6} \tilde{\delta}, \quad \tilde{I} \tilde{\delta}=3 \tilde{c} \int_{0}^{1} \rho_{0} \phi^{2} d y \\
\tilde{I} & =\int_{0}^{1} \rho_{0} \phi_{y}^{2} d y, \quad Q=\frac{2 c^{2} \hat{c}}{H} \tilde{I} .
\end{aligned}
$$

The scaled entities $\tilde{\mu}, \tilde{\delta}, \tilde{I}$ are independent of $x$, and so the dependence of $\mu, \delta, Q$ on $x$ is now explicitly determined. The coefficient $\sigma$ is now given by

$$
\sigma=\frac{\tilde{\sigma}}{2 c_{0} \tilde{c}}, \quad \tilde{I} \tilde{\sigma}=-\int_{0}^{1} \rho_{0 y} \phi \phi_{y}\left[-U^{2} \frac{H_{x}}{H}-g H_{x}(1-y)+g \eta_{0 x}\right] d y,
$$


Using the modal equations $(53,54)$ in the Boussinesq approximation when $\rho_{0}$ in the term $\left(\rho_{0} \phi_{y}\right)_{y}$ is kept constant, this can be expressed as

$$
\begin{aligned}
& \tilde{I} \tilde{\sigma}=\frac{\tilde{c}^{2}}{2}\left\{-J\left(U^{2} \frac{H_{x}}{H}-g \eta_{0 x}\right)-K g H_{x}\right\}, \\
& \tilde{I} J=\left[\rho_{0} \phi_{y}^{2}\right]_{0}^{1}, \quad \tilde{I} K=\left\{\left[\rho_{0}(1-y) \phi_{y}^{2}\right]_{0}^{1}+\tilde{I}\right\} .
\end{aligned}
$$

The sign of $\sigma$ thus depends inter alia on the signs of $J, K$. For a near-surface pycnocline we expect that $J>K>0$ for a mode one wave. Further, in the Boussinesq approximation we expect the term in $g \eta_{0 x}$ can be neglected compared to $g H_{x}$. Assuming here that the waves propagate in the positive $x$-direction when there is no current, that is $\tilde{c}>0$, it follows that $\sigma>0$ when $H_{x}<0$ and $\sigma<0$ when $H_{x}>0$. It follows that there is wave amplification (suppression) when a wave propagates into an decreasing (increasing) current, whether advancing or opposing.

To examine the entities $J, K$ in more detail, we consider a two-layer fluid, $\rho_{0}(y)=\rho_{1} H\left(y-y_{2}\right)+\rho_{2} H\left(-y+y_{2}\right)$ where the upper layer has depth $y_{1}$ and the lower layer has depth $y_{2}$, with $y_{1}+y_{2}=1$. In the Boussinesq approximation,

$$
\phi=\frac{1-y}{y_{1}}, \quad y_{2}<y<1 ; \quad \phi=\frac{y}{y_{2}}, \quad 0<y<y_{2}, \quad \tilde{c}^{2}=y_{1} y_{2} .
$$

In the original variables, the upper layer has depth $h_{1}$ and the lower layer has depth $h_{2}$ where $H=h+\eta_{0}=h_{1}+h_{2}$; hence $y_{1}=h_{1} / H$ and $y_{2}=h_{2} / H$. Note that $y_{1,2}$ are constants, but that $h_{1,2}$ and hence $H$ vary with $x$. Then $J=\left(y_{2}-y_{1}\right) / y_{1} y_{2}$ and $K=\left(y_{2}-y_{1}\right) / y_{2}$. Thus in the usual oceanic situation when the upper layer is thinner than the lower layer, $y_{1}<y_{2}, J>K>0$ as expected. But note that if the upper layer is thicker than the lower layer, these scenarios are reversed.

However, the amplification or suppression of the wave amplitude refers to the amplitude $A$ in the transformed equation (5), and to transfer this result to the physical space amplitude $A=\sqrt{Q} \eta(2)$, it is necessary to evaluate $Q=c^{2} I$ (23) which in this present case reduces to $Q=2 c^{2} \hat{c} \tilde{I} / H$ (57). Thus

$$
\frac{Q_{x}}{Q}=-\frac{3 U H_{x}}{2 c H}, \quad c=c_{0} \tilde{c}+U .
$$

Since we are assuming here that the waves propagate in the positive $c$ direction, $c>0, \tilde{c}>0$, it follows that $Q$ decreases (increases) in the positive 
$x$-direction according as $U H_{x} / H>0(<0)$. Thus, relative to the transformed amplitude $A$, the physical amplitude amplifies when $U H_{x}>0$ and is suppressed when $U H_{x}<0$. That is, there is amplification when the waves propagate into an opposing current of decreasing magnitude $U<0, H_{x}<0$, and when the waves propagate into an advancing current of decreasing magnitude $U>0, H_{x}<0$. This outcome is in complete agreement with the results obtained by Vlasenko and Stashchuk (2006). They used a Boussinesq approximation which lead to a conservative setting for their analysis and numerical simulations. In effect the role of $\sigma$ is excluded from consideration in their work and wave amplification or suppression is controlled by wave action conservation, that is by $Q$. Indeed, it can be show that when the wave-flow interaction equation (6-10) in Vlasenko and Stashchuk (2006) is reduced to the present $\mathrm{vKdV}$ framework, the term $R$ in their equation (10) reduces exactly to $\left(Q_{x} / Q\right) c E$ ( $E$ is their wave energy) with $Q_{x} / Q$ expressed by $(61)$.

\section{Applications II}

In this section we examine the possible effects of a horizontally varying background hydrology using actual ocean data.Specifically, we consider three case studies taken from the papers by Grimshaw et al. (2004, 2006). These are the North West shelf (NWS) of Australia, the Malin shelf edge (MSE) of Scotland and the Arctic shelf (AC) of Russia. These cases were chosen to represent three contrasting scenarios; a shelf (NWS) with considerable variability and a polarity change; a shelf (MSE) with steeper topography leading to non-adiabatic behaviour and fissioning; and a shelf (AS) with smooth topography and adiabatic behaviour. In each case they used orceanic data, including variable background hydrology but not background currents, to calculate the coefficients of the vKdV equation (1) for a mode 1 wave, and then used this equation to simulate the propagation of an internal solitary wave propagating on the continental slope. However, they did not include the term with coefficient $\sigma$ and that omission is remedied here.

Although the effects of the Earth's background rotation are potentially important for the long-time evolution of an internal solitary wave, we have chosen not to consider the effects of rotation in this present work. Our main purpose here is the re-examine the three case studies of Grimshaw et al. (2004) when the "new" non-conservative term $\sigma \eta$ is included. The effects of rotation were not considered in Grimshaw et al. (2004) and to have included 
rotation here would have clouded the issue of the effect of the term $\sigma \eta$. Also, a time scale of $20 h$, albeit greater than an inertial period, is rather too short to see significant effects due to rotation such as the transformation of an internal solitary wave into an envelope wave packet as described by Grimshaw and Helfrich (2008). Our experience is that several inertial periods are needed for this to occur, see for instance the numerical study by Grimshaw et al. (2014) of propagation of an internal solitary wave over a transect of the South China Sea, where using both a variable-coefficient Ostrovsky equation and the MITgcm, it was found that rotation effects started to became evident only after two to three inertial periods. However, we note that the influence of earth's rotation may be important on shorter time scales when a solitary wave train is formed out of an initial pulse, see Gerkema and Zimmerman (1995).

\subsection{North West shelf}

For our purposes we need analytical expressions for the coefficients. From the data shown in figure 3 of the paper by Grimshaw et al. (2004) (see also figure 5 in the paper by Grimshaw et al. (2006)), we set $\mu=(2.5+$ $3 \tanh (0.1 x-5.0) / 100), \delta=12 \exp (-0.04 x)$ and $c=1.4-x / x_{\text {end }}\left(x_{\text {end }}\right.$ is the length of our computational domain) as smooth approximations to the values exhibited by Grimshaw et al. (2004). Note that $\mu, \delta$ are $\alpha, \beta$ respectively in the paper by Grimshaw et al. (2004). The depth $h$ was represented by a polynomial interpolation of the actual depth at five points in the domain $0<x<x_{\text {end }}$.

However, the data obtained from the paper by Grimshaw et al. (2004) is not sufficient to find an analytical expression for $\sigma$. So for $\sigma$ only, we use the two-layer model coefficients (45) and note that then

$$
\mu \delta=\frac{c^{2}}{4}\left(h_{1}-h_{2}\right)=\frac{c^{2}}{4}\left(2 h_{1}-h\right),
$$

so that with $\mu, \delta, c, h$ known, $h_{1}$ can be estimated. With $h_{1}$ found, the known expression for $c$ can be used to find $g^{\prime}$. Then we use the derived $h_{1}, g^{\prime}$ to estimate $\sigma$ from (46).

The final step is to express the non-dimensional $\alpha, \beta$ in (6) as functions of $\tau$. To this end we note that we now have $\alpha=\alpha(x)$, and also $\tau=\tau(x)$ from evaluating the integral in (6). This is a parametric representation of $\alpha=\alpha(\tau)$. If the total $x$-domain is $0<x<x_{\text {end }}$, then the $\tau$-domain is 
$0<\tau<\tau_{\text {end }}$ where

$$
\tau_{\text {end }}=\tau\left(x_{\text {end }}\right)=\int_{0}^{x_{\text {end }}} \frac{\delta}{c^{4}} d x .
$$

Then we obtain a plot of $\alpha(\tau)$ from this parametric representation over the domain $0<\tau<\tau_{\text {end }}$, and from the plot obtain an analytical expression for $\alpha(\tau)$ by a smooth interpolation. The outcome is shown in figure 3.

The numerical simulations based on the vKdV equation (5) are shown in figure 4 for both $\beta=0, \beta \neq 0$ for the initial condition of a solitary wave (32) of amplitude $5 \mathrm{~m}$. The former case corresponds to that in figure 5 of Grimshaw et al. (2004), see also figure 6 of Grimshaw et al. (2006). Note that in our simulations the output is $A(X, \tau)$, and is plotted as a function of $X$ at certain fixed $\tau$. This needs to be interpreted in terms of the original $x, t$ variables through the transformations $(2,6)$ to plot $\eta(x, t)$ as a function of $t$ for certain fixed $x$ as in the figures in Grimshaw et al. $(2004,2006)$. Note that the plots we produce here are also plots as a function of time $t$ (strictly $-t$ ) at certain fixed locations $x_{n}$ determined by $\tau=\tau_{n}=\tau\left(x_{n}\right)$, and $A$ differs from $\eta$ only through the factor $Q^{1 / 2}$, which can alter the magnitude, but not the shape of the wave.

The coefficient $\mu$ of the quadratic nonlinear term is initially negative and then becomes positive, so this is a case with a polarity change. Note that our analytical expression for $\mu$ is smoother in the positive regime, than that displayed in Grimshaw et al. (2004, 2006) but the overall qualitative shape is the same. The dynamics is controlled by the derived coefficients $\alpha, \beta$ and evolution is with resepct to the transformed variable $\tau$ (6). Here, from figure 3 we see that $\alpha$ is initially negative, then becomes positive but quite small before significantly increasing in magnitude. Hence when $\beta=0$, the simulation shown shown in the left column of figure 4 , we see the initial internal solitary wave undergoing the well-known transformation due to the polarity change, that is the replacement of the initial depression wave by a depression rarefaction wave, and the development of elevation solitary waves riding on that pedestal, see Grimshaw and Yuan (2016) and references therein. However, from figure 3 we see that $\beta$ is initially positive indicating wave decay, but then becomes negative and although rather small, will generate wave growth. The effect can seen in the plot for the scaling factor $R(9)$ which is initially slightly less than 1 with a minimum of 0.98 , but then significantly increases above 1 to 1.52 towards the end of the domain. The simulation shown in the right panel of figure 4 for $\beta \neq 0$ indicates that consequently 
the wave develops as for the case $\beta=0$ but with a slightly increased amplitude until the end of the domain, when the elevation waves riding on the depression rarefaction are significantly larger. Note that the magnification factor $Q>1$ everywhere, with the smallest $Q=1$ at $\tau=0$ and the largest $Q=2.82$ at the end of the domain. The consequent decrease from $A$ to $\eta$ is can be seen clearly in Fig. 4, but note that the factor $\sqrt{Q}$ only affects the magnitude and not the fissioning dynamics.

\subsection{Malin shelf edge}

Here we use the same approach for the Malin shelf edge (MSE), using the data shown in figure 7 of Grimshaw et al. (2004) to obtain analytical expressions for the coefficients. Here we setc $=0.75-\left(0.35 x / x_{\text {end }}\right)$, $\delta=36 \exp (-0.04 x)$ and $h=925 \exp (-0.15 x)+200$, while for $\mu$, which corresponds to $\alpha$ in Grimshaw et al. (2004), is represented by a polynomial interpolation of the actual values at five points in the domain $0<x<x_{\text {end }}$. These are plotted in figure 5 together with the derived values of $\alpha$ and $\beta$. In this case there is no polarity change and $\mu$ is negative over the whole domain. $\sigma$ is initially positive, but decreases and eventually becomes slightly negative. However, the scaling factor $R(9)$ is less than 1 over the whole domain, with a smallest value of 0.786 , and then increases towards unity at the end of the domain.

The simulations are shown in figure 6 . When $\beta=0$, the initial solitary wave steepens on the front face, and begins to fission at the end of the domain. But, in contrast to the NWS case, here when $\beta \neq 0, R<1$ over the whole domain, this reducing slightly the effect of nonlinearity sufficiently to inhibit the fissioning at the end of the obtain. The transformation $A=\sqrt{Q} \eta$ (2) to the physical variable $\eta$ shows that initially $\eta$ is enhanced above $A$ as initially $Q<1$ and decreases to a minimum of 0.7 , but then increases to 1.1 and so at the end of the domain $A$ and $\eta$ are virtually the same.

\subsection{Arctic shelf}

For the Arctic shelf, we use the data shown in figure 11 of Grimshaw et al. (2004). Here we set $c=0.84-\left(0.14 x / x_{\text {end }}\right), h=40-\left(22 x / x_{\text {end }}\right)$ and $\delta=55-\left(43 x / x_{e n d}\right)$, and then again use a polynomial approximation for $\mu$. These are plotted in figure 7 together with the derived values of $\alpha$ and $\beta$. The linear long wave speed $c$ decreases only slightly from 0.84 to $0.70 \mathrm{~ms}^{-1}$, and there is then only a small variation of the linear amplification ratio $Q$ of order $10 \%$. The depth also decreases quite slowly over the total distance 
of $160 \mathrm{~km}$. The quadratic nonlinear term $\mu$ does vary significantly, but is everywhere negative, except in the last $5 \mathrm{~km}$, where is a change in polarity at a distance of $155 \mathrm{~km}$. In this case $\beta$ is quite small, and the scaling parameter $R$ does not change much, as the smallest value is $R=1$ while the largest value is only $R=1.02$.

The simulations are shown in figure 8 . When $\beta=0$ the initial solitary wave deforms adiabatically until near the end of the domain, when there is a polarity change, and the wave field transforms to a leading rarefaction wave on which are riding several elevation solitary waves. Here, since $R \approx 1$, the case $\beta \neq 0$ is almost the same. Further because $Q$ does not change very much from 1 , there is very little difference between $A$ and $\eta$.

\section{Discussion}

In this paper we have examined the effect of the nonconservative term $\sigma \eta$ in the $\mathrm{vKdV}$ equation (1), which has hitherto been usually neglected in the many studies using this equation to model the propagation and deformation of oceanic internal solitary waves. We used an analytical two-layer model in section 3 and re-examined three oceanic case studies taken from Grimshaw et al. $(2004,2006)$ in section 4 . These cases were chosen to represent three contrasting scenarios; a shelf (NWS) with considerable variability and a polarity change; a shelf (MSE) with steeper topography leading to non-adiabatic behaviour and fissioning; and a shelf (AS) with smooth topography and adiabatic behaviour. In each case Grimshaw et al. (2004, 2006) used oceanic data, including variable background hydrology but not background currents, to calculate the coefficients of the vKdV equation (1) for a mode 1 wave, and then used this equation to simulate the propagation of an internal solitary wave propagating on the continental slope. However, they did not include the term with coefficient $\sigma$ and that omission is remedied here.

The effect of this term is best seen in the transformed equation (5) through the coefficient $\beta=\sigma c^{3} / \delta$ which includes also. the effect of variability in the dispersion coefficient $\delta$. Further the transformation to the equation (9) indicates that the cumulative effect of $\beta$ is best estimated through the scaling parameter $R$. The essential issue is whether $\sigma<0$ or $\sigma>0$, and so $\beta<0$ $(R>1)$ or $\beta>0(R<1)$ indicating wave amplification or decay respectively. The analytical and oceanic examples we have considered here suggest that although $\beta$ may change sign along the path of the wave, the main issue 
is whether $R>1$ or $R<1$ at the end of the propagation path, and so whether or not the wave amplitude has increased or decreases as the wave reaches shallower depth. Although we have only examined a very few, but representative cases, it appears that no general conclusion can be reached and the outcome depends on the specific case being studied. But we note that when the background is a barotropic tidal current, the case studied by Vlasenko and Stashchuk (2006) and discussed theoretically here in section 3.2 , some general conclusions can be reached. We note that a comparison of the cases when $\beta=0$ and $\beta \neq 0$ suggests that the overall structure of the dynamical wave evolution remains basically the same, and the role of $\beta$ and hence $R$ is mainly to change the wave amplitude.

Since we have demonstrated that the new term $\sigma \eta$ in (1) may be quite significant in practice, it becomes important to consider the origin of the horizontal variation in the background hydrology. In the derivation of this term, the coefficient $\sigma$ expressed in (23) depends on the body force $F_{0}$. This horizontal body force $F_{0}(z ; x)$ represents terms not present in the left-hand side of (10) such as diabatic effects, for instance dissipation, Corlolis terms, and driving terms, for instance wind stress. In this paper, we do not examine this issue directly. Instead, we use model or observed background fields $\rho_{0}(z ; x)$ and $u_{0}(z ; x)$ to estimate the body force $F_{0}(z ; x)$ directly, without regard to its precise physical meaning, and hence find the coefficient $\sigma$. However, in an ongoing study we are examining climatological data to investigate further the physical meaning of the term $\sigma \eta$.

\section{Acknowledgements}

RG was supported by the Leverhulme Trust through the award of a Leverhulme Emeritus Fellowship.

\section{References}

Andrews, D. G., McIntyre, M. E., 1978. On wave-action and its relatives. J. Fluid Mech. 89, 647-664.

Benjamin, T. B., 1966. Internal waves of finite amplitude and permanent form. J. Fluid Mech 25, 241-270.

Benney, D. J., 1966. Long non-linear waves in fluid flows. J. Math. Phys 45, $52-63$. 
El, G., Grimshaw, R., Tiong, W., 2012. Transformation of a shoaling undular bore. J. Fluid Mech. 709, 371-395.

Gerkema, T., Zimmerman, J. T. F., 1995. Generation of nonlinear internal tides and solitary waves. Journal of Physical Oceanography 25 (6), 10811094.

Grimshaw, R., 1979. Slowly varying solitary waves i. Korteweg-de Vries equation. Proc. Roy. Soc 368A, 359-375.

Grimshaw, R., 1981. Evolution equations for long nonlinear internal waves in stratified shear flows. Stud. Appl. Math 65, 159-188.

Grimshaw, R., 1984. Wave action and wave-mean flow interaction, with application to stratified shear flows. Ann. Rev. Fluid Mech. 16, 11-44.

Grimshaw, R., 2001. Internal solitary waves. In: Grimshaw, R. (Ed.), Environmental Stratified Flows. Kluwer, Boston, pp. 1-27.

Grimshaw, R., 2007. Internal solitary waves in a variable medium. Gesellschaft fur Angewandte Mathematik 30, 96-109.

Grimshaw, R., 2015. Change of polarity for periodic waves in the variablecoefficient Korteweg-de Vries equation. Stud. Appl. Math. 134, 363-371.

Grimshaw, R., Guo, C., Helfrich, K., Vlasenko, V., 2014. Combined effect of rotation and topography on shoaling oceanic internal solitary waves. J. Phys. Ocean. 44, 1116-1132.

Grimshaw, R., Helfrich, K. R., 2008. Long-time solutions of the Ostrovsky equation. Stud. Appl. Math. 121, 71-88.

Grimshaw, R., Pelinovsky, E., Stepanyants, Y., Talipova, T., 2006. Modelling internal solitary waves on the Australian North West shelf. Marine and Freshwater Research 57, 265.

Grimshaw, R., Pelinovsky, E., Talipova, T., Kurkin, A., 2004. Simulation of the transformation of internal solitary waves on oceanic shelves. J. Phys. Ocean 34, 2774-2779. 
Grimshaw, R., Pelinovsky, E., Talipova, T., Kurkina, A., 2010. Internal solitary waves: propagation, deformation and disintegration. Nonlinear Processes in Geophysics 17, 633-649.

Grimshaw, R., Yuan, C., 2016. The propagation of internal undular bores over variable topography. Physica D 333, 200-207.

Helfrich, K. R., Melville, W. K., 2006. Long nonlinear internal waves. Ann. Rev. Fluid Mechanics 38, 395-425.

Holloway, P., Pelinovsky, E., Talipova, T., 2001. Internal tide transformation and oceanic internal solitary waves. In: Grimshaw, R. (Ed.), Environmental Stratified Flows. Kluwer, Boston, pp. 31-60.

Ostrovsky, L. A., Stepanyants, Y. A., 2005. Internal solitons in laboratory experiments: Comparison with theoretical models. Chaos 28, 037111.

Ott, E., Sudan, R., 1970. Damping of solitary waves. Phys. Fluids 13, 14321434.

Pelinovsky, E. N., Rayevsky, M., Shavratsky, S. K., 1977. The Kortewegde Vries equation for nonstationary internal waves in an inhomogeneous ocean. Izvestiya, Atmospheric and Oceanic Physics 13, 226-228.

Vlasenko, V. I., Stashchuk, N., 2006. Amplification and suppression of internal waves by tides over variable bottom topography. J. Phys. Ocean. 36, 1959-1973.

Vlasenko, V. I., Stashchuk, N. M., Hutter, K., 2005. Baroclinic Tides: Theoretical Modelling and Observational Evidence. Cambridge University Press.

Zhou, X., Grimshaw, R., 1989. The effect of variable currents on internal solitary waves. Dyn. Atmos. Oceans 14, 17-39. 

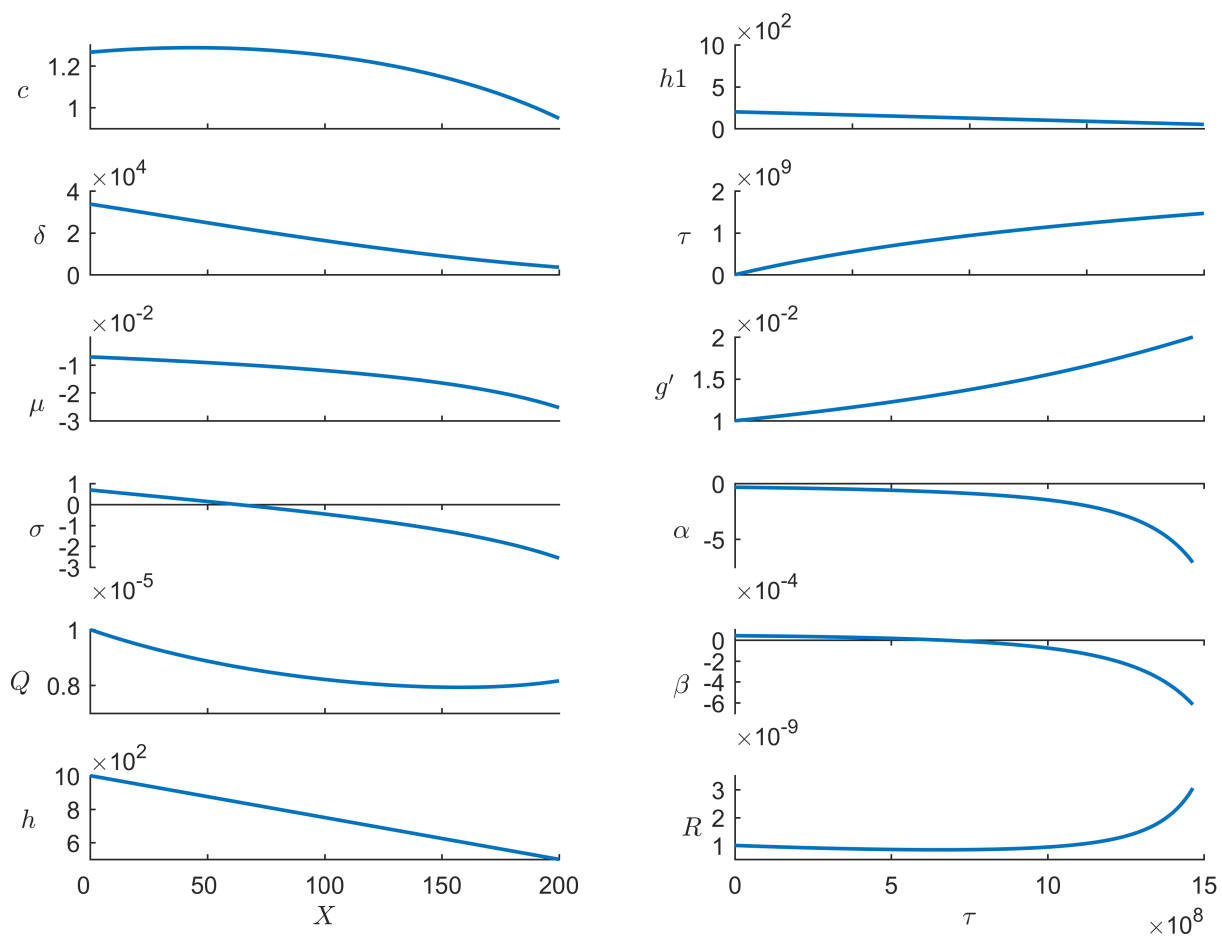

Figure 1: Variation of the coefficients(left) and derived coefficients(right) of the $\mathrm{vKdV}$ equation for a two-layer fluid model. 

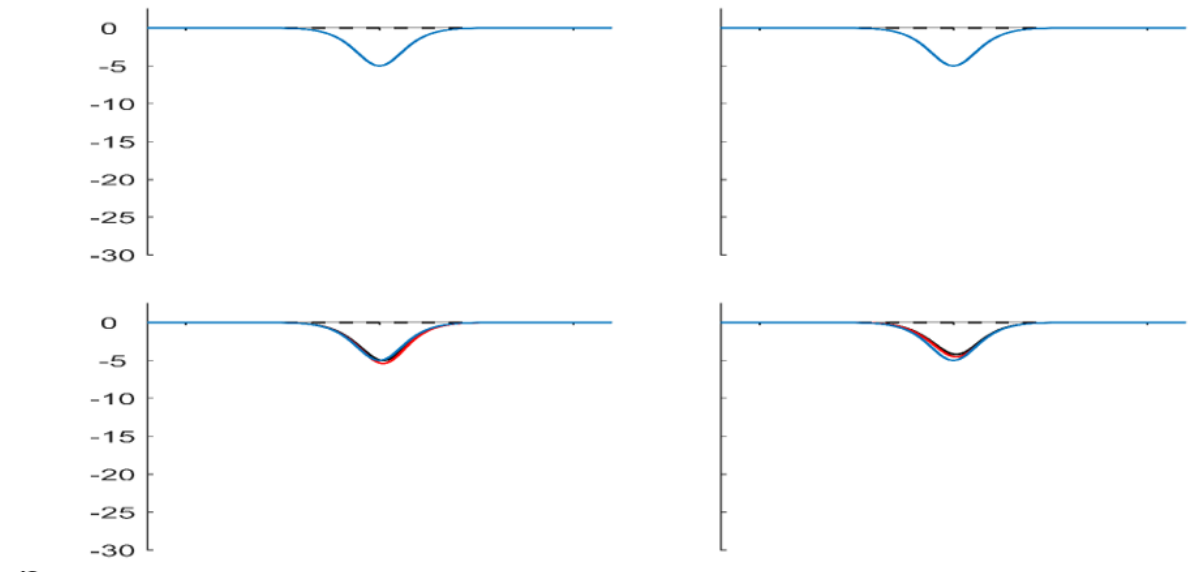

$\eta$
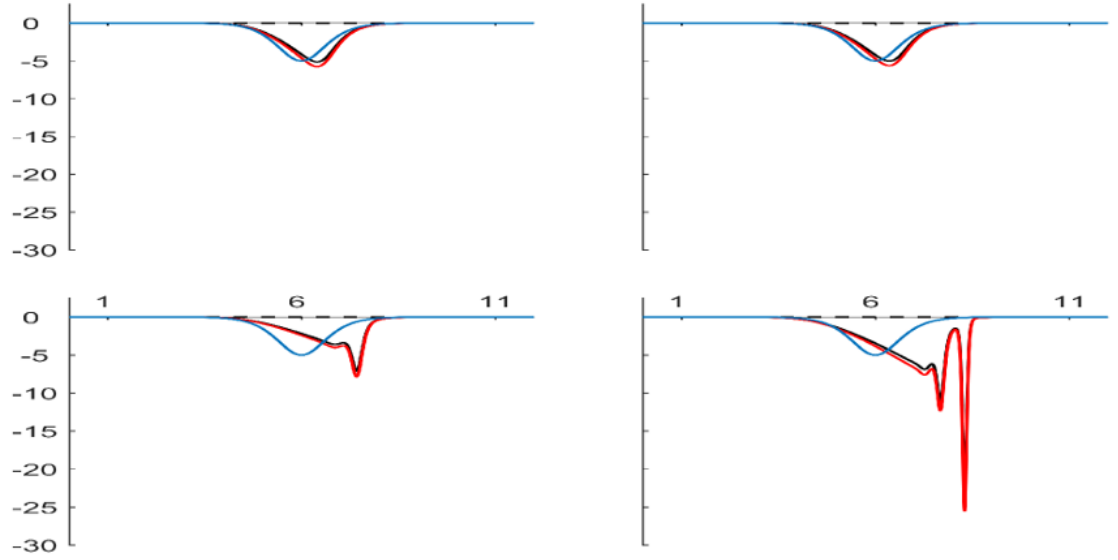

Time

Figure 2: A numerical simulation of the vKdV equation (5) for the two-layer fluid model, for the cases when $\sigma=0$ (right four panels) and $\sigma \neq 0$ (left four panels). The initial condition is the solitary wave (32) (blue), the numerical solution is $A$ (black) and the physical solution is $\eta$ (red). From top to bottom, the distances from the initial point are $0 \mathrm{~km}, 65 \mathrm{~km}, 130 \mathrm{~km}, 200 \mathrm{~km}$. 

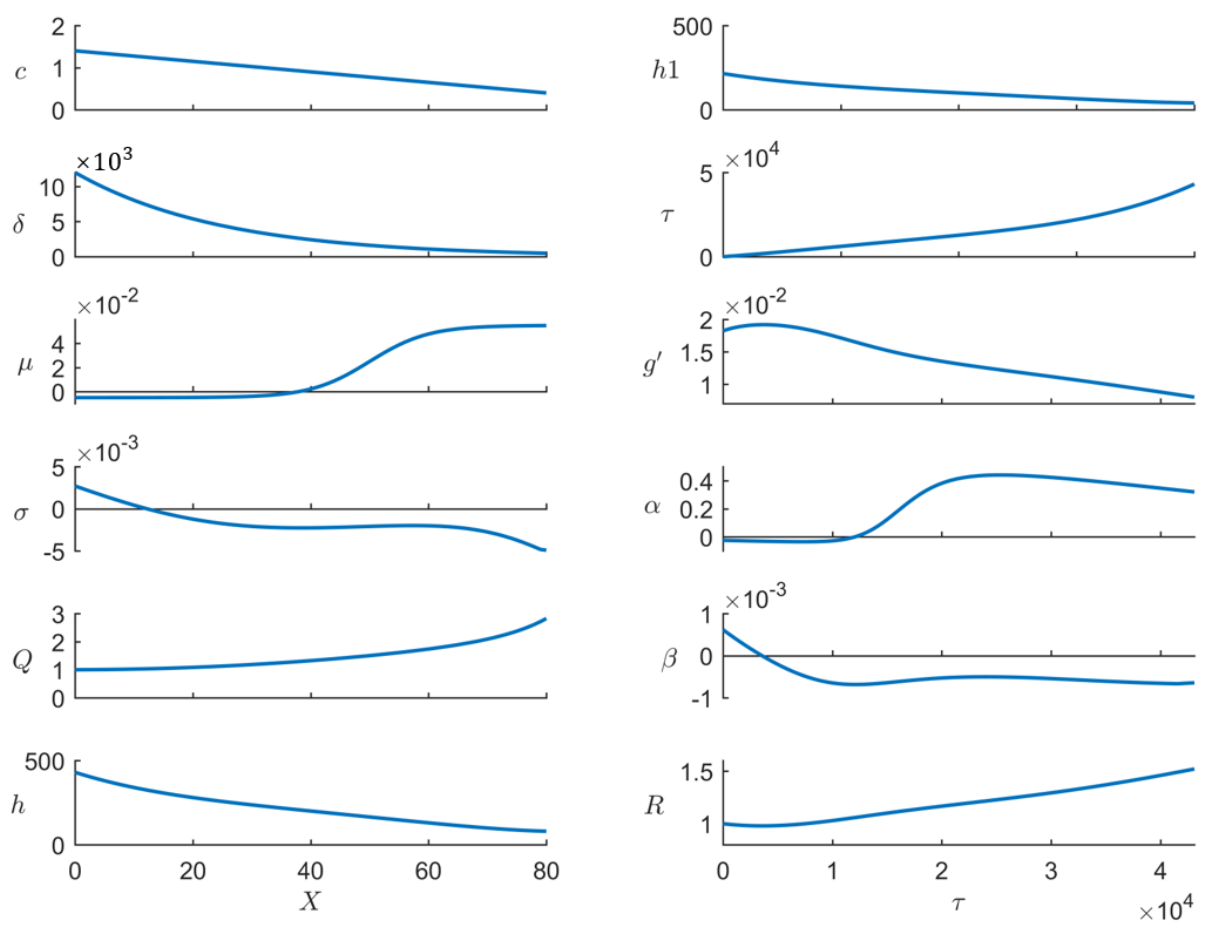

Figure 3: Variation of the estimated coefficients (left) and derived coefficients (right) of the vKdV equation for conditions of the North West Shelf. 

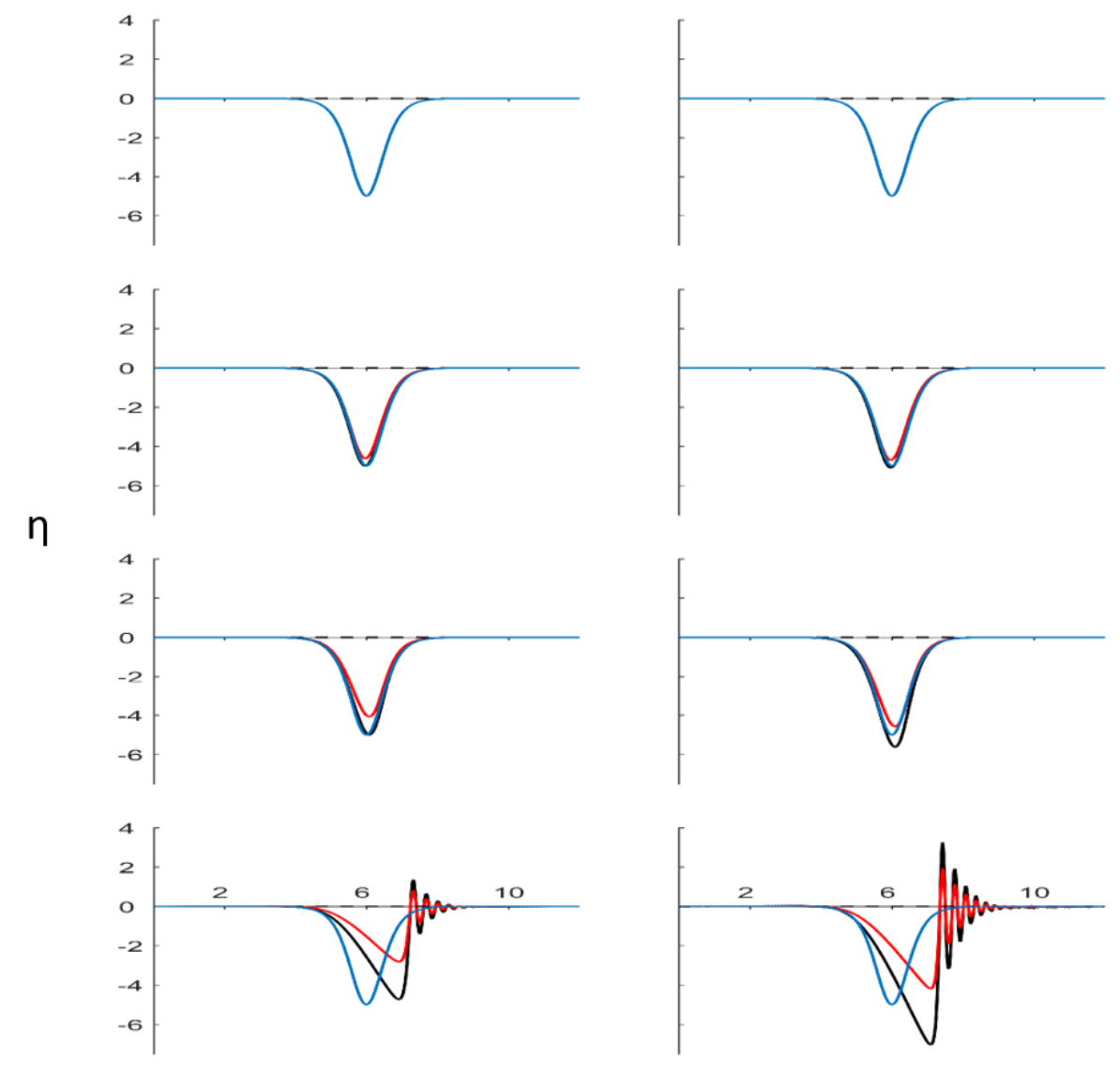

Time

Figure 4: A numerical simulation of the vKdV equation (5) for the North West shelf when $\beta=0$ (left) or $\beta \neq 0$ (right). The initial condition is the solitary wave (32) (blue), the numerical solution is $A$ (black) and the physical solution is $\eta$ (red) of North West shelf. From top to bottom, the distances from the initial point are $0 \mathrm{~km}, 34 \mathrm{~km}, 52 \mathrm{~km}, 74 \mathrm{~km}$. 

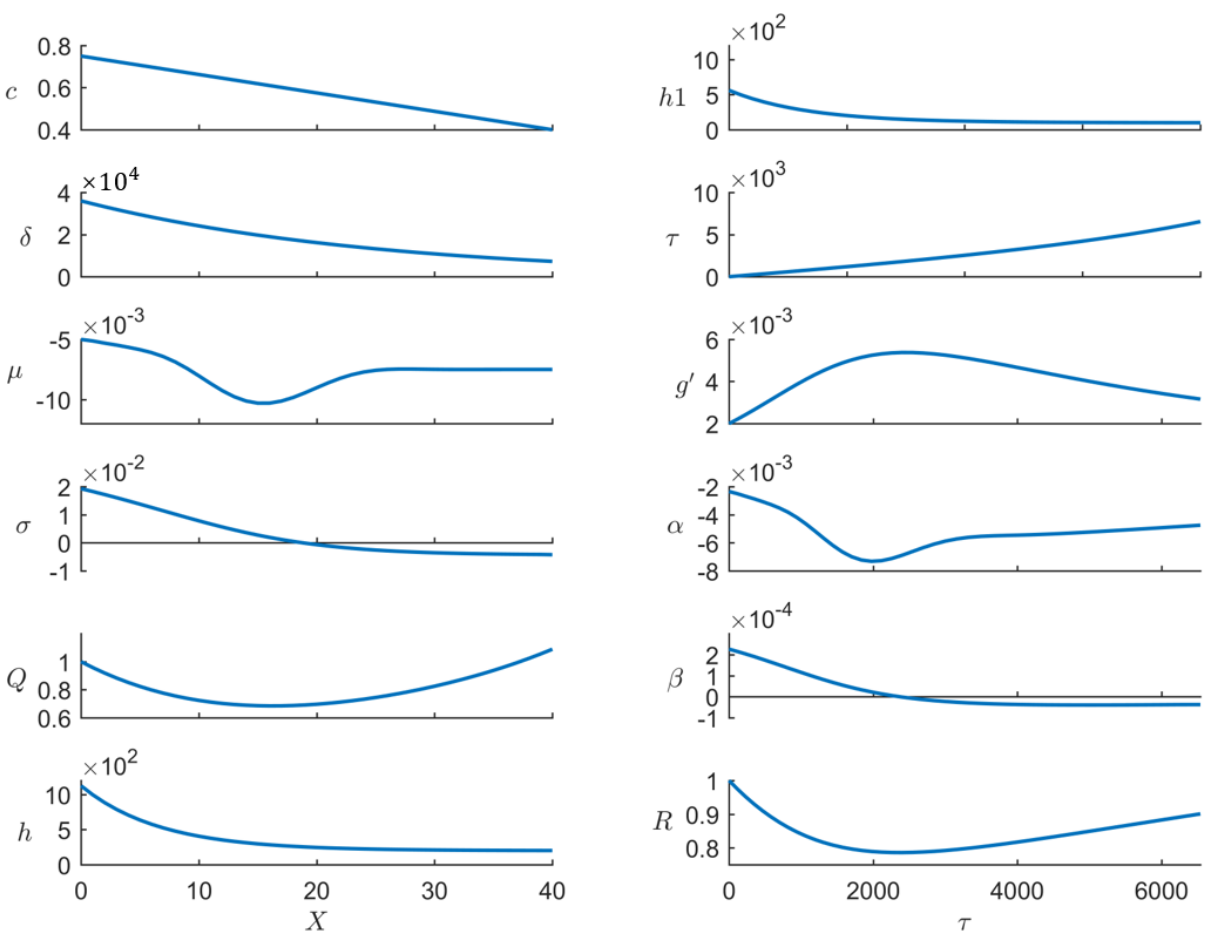

Figure 5: Variation of the estimated coefficients(left) and derived coefficients(right) of the variable-coefficient $\mathrm{KdV}$ equation for conditions of the Malin shelf edge. 

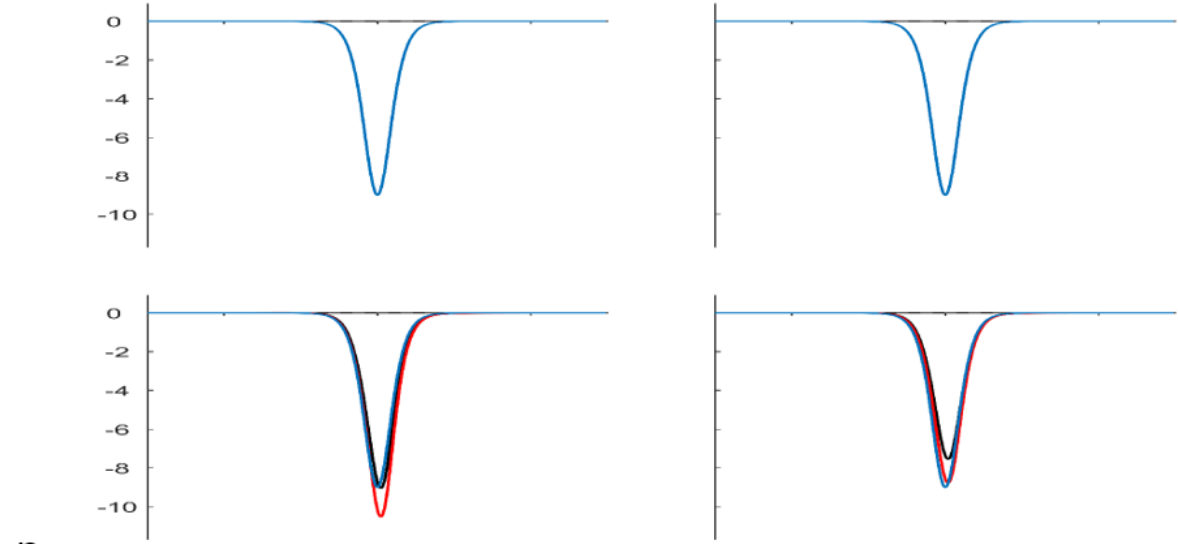

$\eta$
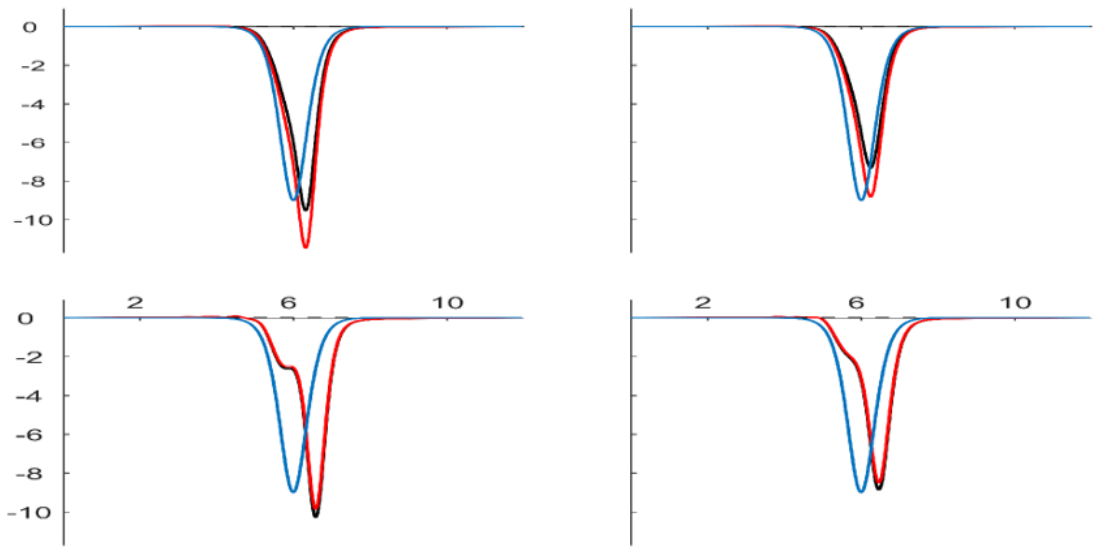

Time

Figure 6: A numerical simulation of the vKdV equation (5) for the Malin Shelf edge when $\beta=0$ (left) and $\beta \neq 0$ (right). The initial condition is the solitary wave (32) (blue), the numerical solution is $A$ (black) and the physical solution is $\eta$ (red) of Malin shelf edge. From top to bottom, the distances from the initial point are $0 \mathrm{~km}, 11 \mathrm{~km}, 21 \mathrm{~km}, 36 \mathrm{~km}$. 

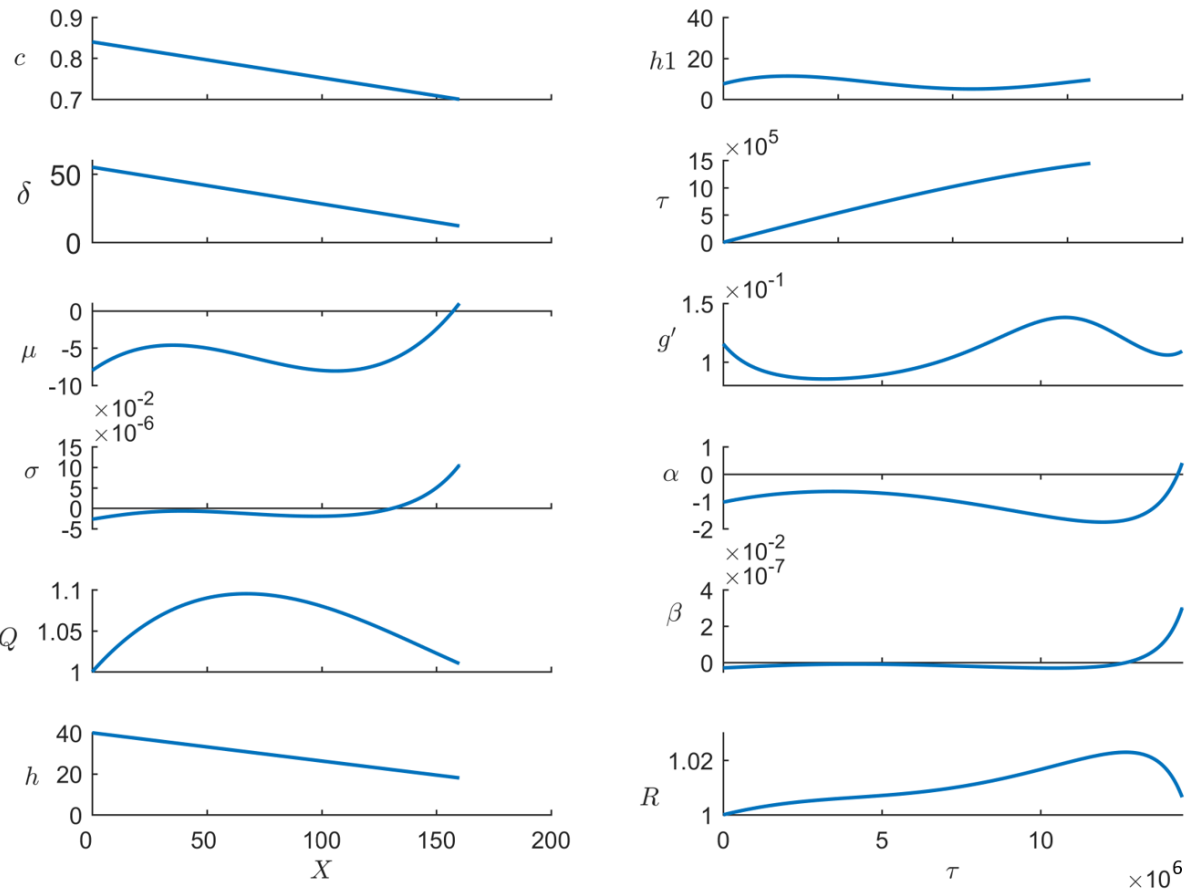

Figure 7: Variation of the estimated coefficients(left) and derived coefficients(right) of the variable-coefficient $\mathrm{KdV}$ equation for conditions of the Arctic shelf. 

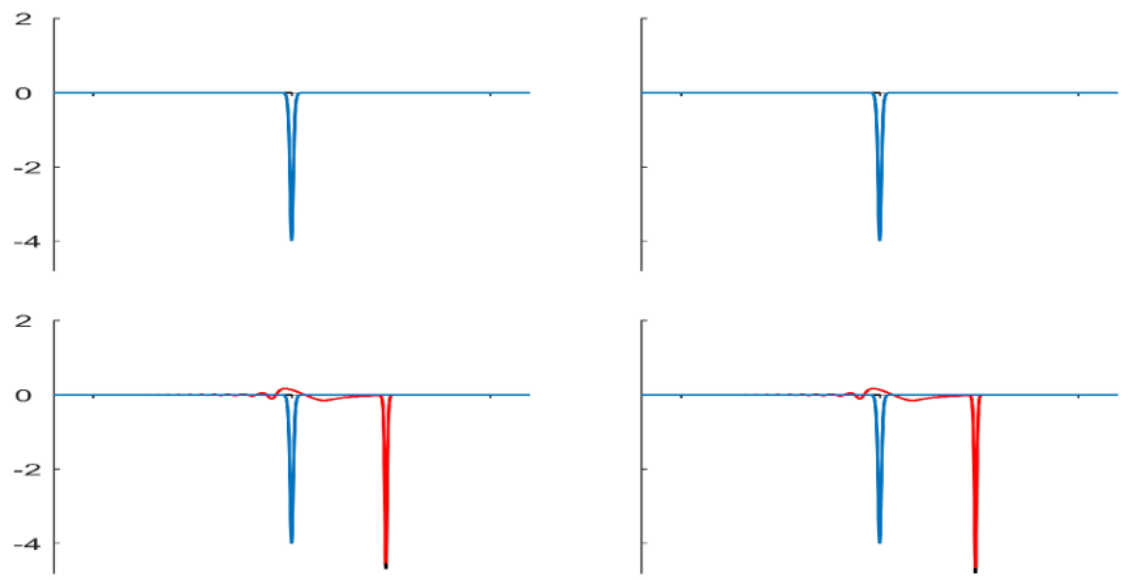

$\eta$
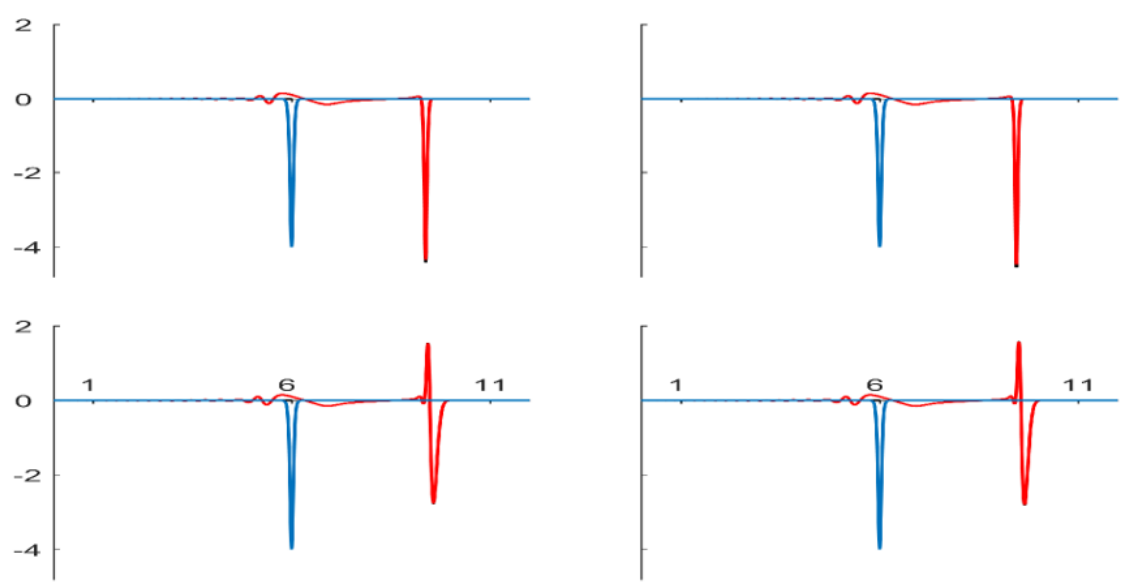

\section{Time}

Figure 8: A numerical simulation of the vKdV equation (5) for the Arctic shelf for cases when $\beta=0$ (left) and $\beta \neq 0$ (right). The initial condition is the solitary wave (32) (blue), the numerical solution is $A$ (black) and the physical solution is $\eta$ (red) of Arctic shelf. From top to bottom, the distances from the initial point are $0 \mathrm{~km}, 118 \mathrm{~km}, 142 \mathrm{~km}, 158 \mathrm{~km}$. 